\title{
A RUNGE THEOREM FOR SUBHARMONIC FUNCTIONS ON CLOSED SUBSETS OF RIEMANNIAN MANIFOLDS
}

\section{PIERRE BLANCHET}

Centre de Recherches Mathématiques

Université de Montréal

C.P. 6128 , Succursale Centre-ville

Montréal (Québec)

Canada H3C 3J7

e-mail: vltcbqmj@yahoo.fr

\begin{abstract}
Let $F$ be a closed subset of a non-compact Riemannian manifold $\Omega$. This article presents a proof that every continuous and subharmonic function on a neighbourhood of $F$ can be uniformly approximated on $F$ by a function, which is $C^{\infty}$ and subharmonic on $\Omega$ except for singularities.
\end{abstract}

\section{Introduction}

In a recent article, the author proved an approximation theorem for subharmonic functions on compact subsets of non-compact Riemannian manifolds ([1]). The purpose of this work is to extend this result to subharmonic functions on closed subsets of non-compact Riemannian manifolds.

2010 Mathematics Subject Classification: 31B05.

Keywords and phrases: subharmonic functions, subharmonic approximation, Newtonian functions, Runge theorem, Riemannian manifolds.

Received January 29, 2015

(ㄷ) 2015 Scientific Advances Publishers 
Let $\Omega$ be an orientable Riemannian manifold and $\Delta$ be the Laplacian of $\Omega$. We say that a continuous function $u: \Omega \rightarrow[-\infty, \infty]$ is Newtonian provided that there is a discrete set $A \subset \Omega$ with the following properties:

(i) $\left.u\right|_{\Omega \backslash A}$ is harmonic, and

(ii) for each point $a \in A$, there is a regular subregion $R$ satisfying $R \cap A=\{a\}$ and a constant $c$, such that the function $u-c G_{R}(\bullet, a)$ can be defined at $a$ so as to be harmonic on $R$, where $G_{R}(\bullet, a)$ is the Green's function of $R$.

We say that a continuous function $u: \Omega \rightarrow[-\infty, \infty]$ is subharmonic singular provided $u=v+w$, where $v$ is Newtonian on $\Omega$ and $w \in C^{\infty}(\Omega)$ is subharmonic on $\Omega$. Thus $\Delta u \geq 0$ on $\Omega$ except possibly for isolated singularities.

Theorem. Let $\Omega$ be a non-compact orientable Riemannian manifold. Let $F$ be a closed subset of $\Omega$, and $f$ be continuous and subharmonic on an open set containing $F$. If $\varepsilon>0$, then there exists a subharmonic singular function $h$ on $\Omega$ such that $\sup _{F}|f-h|<\varepsilon$. Moreover, if $\Omega * \backslash F$ is connected and locally connected, we may take $h \in C^{\infty}(\Omega)$ subharmonic on $\Omega$.

Remarks. As usual, $\Omega *=\Omega \cup\{*\}$ denotes the Alexandroff one-point compactification of $\Omega$ and we refer to the point $*$ as the ideal point for $\Omega$. Therefore, if $f \in C^{0}(\Omega)$ is subharmonic on $\Omega$ and if $F$ is any closed subset of $\Omega$ such that $\Omega * \backslash F$ is connected and locally connected, then given $\varepsilon>0$, it follows that there exists $h$ in $C^{\infty}(\Omega)$ subharmonic in $\Omega$ such that $|f-h|<\varepsilon$ on $F$. 
If $\Omega * \backslash F$ is connected and locally connected, then it is known that there exists $h$ continuous and subharmonic on $\Omega$ such that $|f-h|<\varepsilon$ on $F$, see [2]. The originality of this part of the theorem is the fact that we may take $h$ subharmonic in $C^{\infty}(\Omega)$.

In the proof of our approximation theorem, we shall need the following lemma as a technical tool.

Localization theorem. Let $\Omega$ be an orientable non-compact Riemannian manifold. Let $F$ be a closed subset of $\Omega$, and $u \in C^{0}(F)$. Then $u$ can be uniformly approximated on $F$ by a $C^{\infty}$ subharmonic function defined on an open neighbourhood of $F$, if and only if for each $p \in F$, there is a compact set $K_{p}$ such that $p \in \operatorname{int} K_{p}$ and $u_{\mid F \cap K_{p}}$ can be uniformly approximated on $F \cap K_{p}$ by a $C^{\infty}$ subharmonic function defined on an open neighbourhood of $F \cap K_{p}$.

This follows from the Localization theorem presented in [3, Section 3 , Corollary 2].

Proof of Runge theorem. Let $f$ be a continuous and subharmonic function defined on an open neighbourhood $N$ of the closed subset $F$. Then, according to [4, page 268], $f$ can be uniformly approximated on any compact subset $K$ of $N$ by a $C^{\infty}$ strictly subharmonic function defined on an open neighbourhood of $K$. Therefore, from the Localization theorem, we deduce the fact that $f$ can be uniformly approximated on the whole of $F$ by a $C^{\infty}$ subharmonic function defined on an open neighbourhood of $F$.

Let $U$ be an open neighbourhood of $F$ and let $g: U \rightarrow \mathfrak{R}$ in $C^{\infty}(U)$ be subharmonic on $U$ such that

$$
|f-g|<\varepsilon / 2 \text {, on } F \text {. }
$$


Since $\Omega$ is second countable, there exist partitions of unity subordinate to any open cover of $\Omega$ [5, Theorem 2.2.14]. It follows that there exists a function $\Psi \geq 0$ in $C^{\infty}(\Omega)$ such that $\Psi \equiv 1$ on an open neighbourhood of $F$, and $\Psi \equiv 0$ on an open neighbourhood of $\Omega \backslash U$. Let $u=\Psi \Delta g$, with the understanding that $u \equiv 0$ on $\Omega \backslash U$. Since $u \in C^{\infty}(\Omega)$, it follows from [4, Lemma 1, page 268] that there exists a function $v \in C^{\infty}(\Omega)$ satisfying $\Delta v=u$ on $\Omega$. Since $\Psi \geq 0$ on $\Omega$ and $\Delta g \geq 0$ on $U$, we see that $\Delta v \geq 0$ on $\Omega$ and hence $v$ is subharmonic on $\Omega$. Since $\Delta(g-v)=\Delta g-\Delta v=\Delta g-\Psi \Delta g \equiv 0$ on an open neighbourhood of $F$, we may apply [6, Theorem 5.1] to obtain $v_{1}$ Newtonian on $\Omega$ such that $\left|g-v-v_{1}\right|<\varepsilon / 2$ on $F$. Setting $h=v+v_{1}$, we obtain a subharmonic singular function on $\Omega$ such that

$$
|g-h|<\varepsilon / 2 \text {, on } F \text {. }
$$

The first conclusion of the theorem follows from inequalities (1) and (2). To prove the second conclusion of the theorem, we assume that $\Omega^{*} \backslash F$ is connected and locally connected. Then, we may apply the Runge theorem for global harmonic functions [6, Theorem 9.3] to obtain $w_{1}$ harmonic on $\Omega$ such that $\left|g-v-w_{1}\right|<\varepsilon / 2$ on $F$. Setting $h=v+w_{1}$, we obtain a function in $C^{\infty}(\Omega)$ subharmonic on $\Omega$ such that inequality (2) is satisfied. Then, the second conclusion of the theorem follows from this together with inequality (1).

For more insight into the applicability of this result and other similar theorems, the reader is invited to consult our recent article [7] and the references given there. 


\section{References}

[1] P. Blanchet, A Runge theorem for subharmonic functions on Riemannian manifolds, Complex Variables and Elliptic Equations 53(9) (2008), 817-818.

[2] P. M. Gauthier, Subharmonic extensions and approximations, Can. Math. Bull. 37 (1994), 46-53.

[3] P. M. Gauthier, Approximation by (pluri) subharmonic functions: Fusion and localization, Can. J. Math. 44(5) (1992), 941-950.

[4] R. E. Greene and H. Wu, Integrals of subharmonic functions on manifolds of nonnegative curvature, Inv. Math. 27 (1974), 265-298.

[5] R. Narasimhan, Analysis on Real and Complex Manifolds, 3rd Edition, NorthHolland, Amsterdam, 1985.

[6] T. Bagby and P. Blanchet, Uniform harmonic approximation on Riemannian manifolds, J. Anal. Math. 62 (1994), 47-76.

[7] P. Blanchet, Carleman approximation on Riemannian manifolds by harmonic functions with Newtonian singularities, American Journal of Applied Mathematics 3(1) (2015), 1-3. 